\title{
The Epidemiologic Evidence and Potential Biological Mechanisms for a Protective Effect of Dietary Fiber on the Risk of Colorectal Cancer
}

\author{
Rachel R. Huxley • Mark Woodward • Peter Clifton
}

Published online: 25 October 2012

(C) Springer Science+Business Media New York 2012

\begin{abstract}
Cancers of the colon and rectum represent a significant cause of morbidity and mortality worldwide with the burden especially high in North America, Europe, and in some parts of Asia. More than half of the disease burden has been attributed to an inappropriate diet and lifestyle. Low intakes of dietary fiber are considered to be a risk factor for colorectal cancer, although the epidemiological evidence until now has been conflicting in part due to the difficulties in reliably examining the relationships between components of the diet and disease outcomes due to bias, confounding, and measurement error. Results from recently published, large, prospective, cohort studies and from a meta-analysis of the evidence provide "convincing" evidence of an independent dose-response relationship between total dietary fiber intake and increasing risk of colorectal cancer. The anticarcinogenic properties of fiber on cancers of the colon and rectum, however, have still to be elucidated.
\end{abstract}

Keywords Epidemiology $\cdot$ Dietary fiber $\cdot$ Colorectal cancer $\cdot$ Biological mechanisms

\footnotetext{
R. R. Huxley $(\bowtie)$

Division of Epidemiology and Community Health,

University of Minnesota,

$1300 \mathrm{~S}$ 2nd St, suite 300,

Minneapolis, MN 55454, USA

e-mail: rhuxley@umn.edu

R. R. Huxley $\cdot$ M. Woodward

The George Institute for Global Health, University of Sydney,

Sydney, New South Wales, Australia

P. Clifton

Baker IDI Heart and Diabetes Institute,

Melbourne, Victoria, Australia
}

\section{Introduction}

Combined, cancers of the colon and rectum (colorectal) constitute approximately $10 \%$ of all cancers worldwide, and more than half a million people die from colorectal cancer annually-equivalent to approximately $8 \%$ of all cancer-related deaths worldwide [1]. The burden of this disease is particularly high in developed countries where these malignancies rank second in terms of both incidence and mortality compared with approximately fifth in lowerand middle-income countries [1]. The occurrence of colorectal cancer varies at least 25 -fold between countries [1, 2] with the highest incidence rates for colorectal cancer seen in certain areas and ethnic groups in the United States, Canada, Japan, and New Zealand and the lowest rates in South East Asia and Africa [3]. It is this wide geographical variation in incidence rates for colorectal cancer, combined with data from migrant studies [4] that strongly suggest that modifiable risk factors, including diet $[5 \cdot, 6,7]$, physical activity $[5 \cdot 8]$, obesity $[5 \cdot, 9]$, cigarette smoking, and diabetes $[5 \bullet$, $10]$, play a pivotal role in the aetiology of this malignancy [5•, 6-11] (Fig. 1). Indeed, an inappropriate diet, combined with physical inactivity, has been estimated to explain more than half of the disease burden for colorectal cancer [1].

With respect to diet, the components that are considered to have a causative role in the disease, and for which there is the strongest evidence, include high intakes of red and processed meat and high alcohol consumption, whereas intake of dietary fiber is suggested to have a protective effect $[5 \cdot, 12]$. However, the magnitude of these associations is generally weak (Fig. 1), which often has hindered their detection in epidemiological studies where confounding, measurement error, and other forms of bias can drown out 


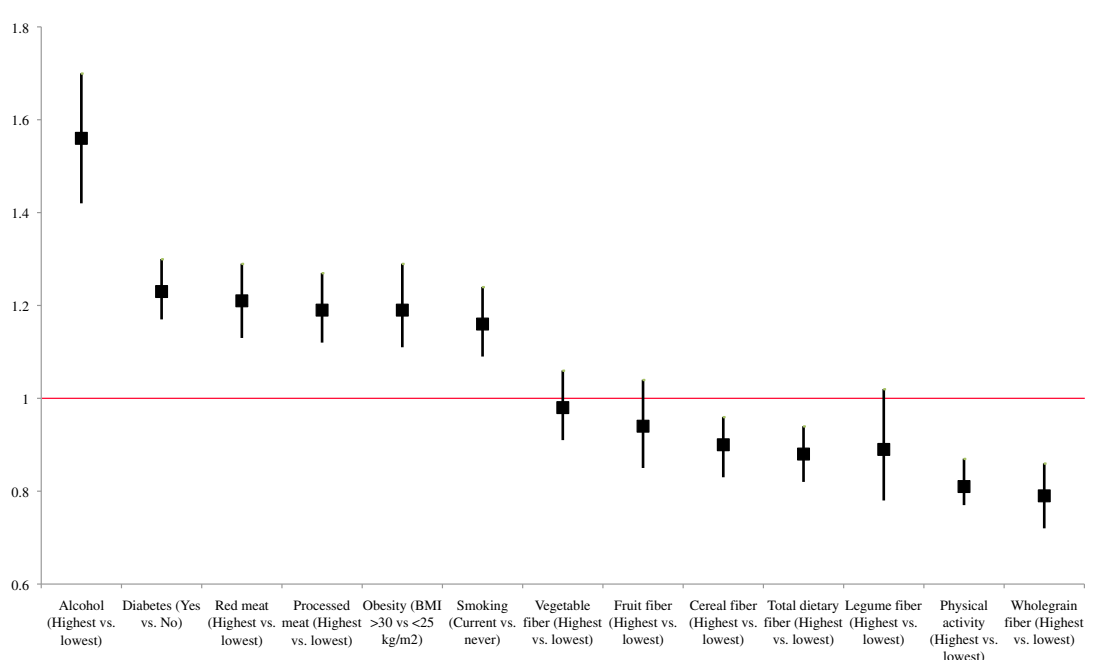

Fig. 1 Relationships between dietary and lifestyle risk factors and risk of incident colorectal cancer as reported in two published metaanalyses from Huxley et al. [5•] and Aune et al. [24••]. (Black square) pooled estimates of effect size; (vertical line) $95 \%$ confidence intervals for the observed effect in the pooled estimate. The horizontal line

any underlying signal. Therefore, the relationships between an exposure of interest and disease outcome often only become apparent in very large, well-conducted cohort studies (such as the European Prospective Investigation into Cancer and Nutrition [EPIC]) [13] or meta-analyses of cohort studies. However, even then there may still be insufficient statistical power to observe a significant association (e.g., in the Pooling Project [14]) if the signal to noise ratio is low.

Moreover, examining the relationship between dietary fiber and risk of colorectal cancer in epidemiological studies is beset by several other important limitations. First, dietary fiber is a term that comprises a wide variety of plant matter, including non-starch polysaccharide (NSP), resistant starch, inulin, lignin, waxes, chitins, pectins, beta-glucans, and oligosaccharides - not all of which are measured in cohort studies and which may each have differing effects on the risk of disease [15]. Second, individuals who have diets that are high in fiber also tend to have other healthy behaviors, such as consuming relatively low amounts of saturated fat, red and processed meat, and alcohol. Therefore, in epidemiological studies it often is very difficult, if not impossible, to completely disentangle the effect of diets that are rich in fiber from other healthy diet and lifestyle behaviors, resulting in residual confounding.

The first part of this review describes the most recent epidemiological advances pertaining to the role of dietary fiber on subsequent risk of cancers of the colon and rectum, which for the purposes of this review are considered collectively as colorectal cancer. The second part details recent advances in understanding possible biological mechanisms by which dietary fiber impacts the risk of developing this malignancy. represents unity. The comparison groups are shown for each of the diet and lifestyle variables assessed; for alcohol, physical activity, red and processed meat, and for all sources of fiber intake the comparison was for the highest compared with the lowest intake. BMI, body mass index

\section{Recent Advances in the Epidemiological Evidence}

In 2007, the World Cancer Research Fund (WCRF) [12] released the Second Expert Report summarizing the evidence for the role of diet and lifestyle risk factors on risk of cancers of the colon and rectum. This report concluded that there was "probable" evidence for a protective effect of foods containing dietary fiber on risk of colorectal cancer, based largely on a pooled analysis of 13 prospective cohort studies with information from more than 725,000 individuals and 8,000 cases of colorectal cancer from North America and Europe [14]. In that analysis, there was a significant inverse association between fiber intake and risk of colorectal cancer in the age-adjusted model (pooled relative risk $(\mathrm{RR})=0.84 ; 95 \%$ confidence interval $(\mathrm{CI}), 0.77-0.92)$, but once consideration was made for relevant confounders, including other dietary components, body mass index (BMI), smoking, and sociodemographic characteristics, the association was attenuated and no longer statistically significant: pooled RR $=0.94 ; 95 \%$ CI, 0.86-1.03.

Since the WCRF report [12], several other, large, prospective, cohort studies have been published that evaluated the relationship between dietary fiber and risk of colorectal cancer [16-23]. In 2011, all of the available data from published cohort studies that had reported on the relationship between dietary fiber and colorectal cancer were combined in a meta-analysis [24••]. This overview included data from 25 prospective cohort studies from the United States, Europe, and Asia that were conducted within the past 25 years, with information on nearly two million individuals and more than 14,000 cases of colorectal cancer, making it the largest dataset on this topic. In that review, Aune and 
colleagues reported that of the 19 studies (most of which had adjusted for relevant confounders) reporting on the relationship by high versus low total dietary fiber intake, those individuals with the highest intakes had a statistically significant $12 \%(95 \% \mathrm{CI}, 6-18)$ reduction in the risk of colorectal cancer compared with those with the lowest intakes. Furthermore, there was no evidence of important heterogeneity between studies (i.e., estimate of effect was broadly consistent across different studies). A total of 16 studies with information on nearly two million individuals and more than 14,000 cases of the neoplasm contributed to a dose-response analysis, which showed that for every $10 \mathrm{~g} / \mathrm{day}$ of total dietary fiber intake, the risk of colorectal cancer significantly decreased by $10 \%(95 \%$ CI, 6-14) [24••].

A range of sensitivity analyses was conducted in an attempt to ascertain whether the positive association with colorectal cancer was present for all sources of dietary fiber. The authors examined the relationship between fruit, vegetables, legumes, cereal, and whole grains and reported that there was only statistically significant evidence of a protective association for fiber derived from cereals (pooled RR per $10 \mathrm{~g}$ of cereal fiber: $0.9 ; 95 \% \mathrm{CI}, 0.83-0.97$ ) and from an extra three servings daily of whole grains $(90 \mathrm{~g})$ (pooled RR, 0.83; $95 \%$ CI, 0.78-0.89). Because some of these analyses were based on data from only four studies, the lack of a significant association with some sources of dietary fiber may be due more to measurement error and associated noise than any true lack of a relationship [24••].

Shortly after the updated meta-analysis by Aune and colleagues was published $[24 \cdot \bullet]$, updated findings of the association between fiber intake and risk of cancers of the colon and rectum from EPIC - a large multicenter cohort study of more than half a million individuals - appeared. These were based on a much longer duration of follow-up than in the previous EPIC publication that was included in the review by Aune and colleagues (11 yr vs. 6 yr) and thus many more incident cases $(4,517$ vs. 1,721$)$ [25•]. Importantly, 23 of the EPIC study centers used the same standardized methodology to examine relationships between exposures and outcomes and hence they were able to correct partially for the effect of dietary assessment measurement error through regression calibration. The key findings from this study were in line with those of the meta-analysis by Aune and colleagues [24.०] and provided more evidence of a modest and independent dose-response relationship between dietary fiber and risk of colorectal cancer; in the model that was adjusted for a range of risk factors and confounders, including total energy intake, BMI, physical activity, and alcohol and meat intake, each $10 \mathrm{~g}$ /day increase in fiber was associated with a significant $13 \%(95 \%$ CI, 4 $21)$ reduction in the risk of colorectal cancer. Moreover, for colon cancer the protective effect of dietary fiber was apparent for all sources of fiber (cereals, fruits, and vegetables), whereas for rectal cancer, only fiber from cereals was significantly associated with reduced risk.

In 2011, the WCRF's Second Expert Report [12] was updated and revised to account for the accumulating evidence for a protective effect of dietary fiber on risk of colorectal cancer. From there being only "possible" evidence of an association in 2007, the association was now considered to be "convincing," although the group noted that evidence gaps still remain pertaining to the impact of fiber intake on colorectal subsites and the relationship between sources of dietary fiber with cancer risk [26••]. Although not directly comparable, the size of the inverse association between total dietary fiber intake (particularly from wholegrains) and risk of developing colorectal cancer is similar to that for physical activity - the only other modifiable risk factor that is considered to be protective against this malignancy (Fig. 1).

\section{Potential Biological Mechanisms for Fiber Protection} against Colonic Cancer

Numerous biological mechanisms have been suggested to explain the anticarcinogenic properties of fiber, including decreased bowel transit times, dilution of fecal carcinogens, increased phenolic acids, lowering of fecal $\mathrm{pH}$ (which leads to lower absorption of carcinogens), increased butyrate production, and decreased ammonia, phenols, and indoles [27]. Many of these observations were made many years ago, and only the most recent observations will be reviewed in detail here. The most active area of research is concerned with defining the microbiota (i.e., the bacterial populations living in the human gut) in normal and diseased states and the effect of diet (including fiber) on the microbiota. In recent years, there has been accumulating interest about the possible role that the gut microbiome may have in the etiology of certain diseases, such as irritable bowel disease, Crohn's disease, diabetes, and even obesity [28]. We concentrate on the evidence regarding a possible relationship between the microbiome and carcionogenesis.

\section{Impact of Diet on the Microbiome}

Human feces are genotoxic and cytotoxic to colon cells [29, 30]. Studies in germ-free mice have shown that fewer cancers are formed with chemical carcinogens compared with mice with normal faecal bacteria [31]. Bacteria are capable of forming genotoxic products from normal dietary components, especially meat protein [32]. Information regarding the species and functional composition of the human gut microbiome is limited to data from a handful of cohorts with little evidence regarding variation across the world. Arumugam and colleagues performed the equivalent of a meta-analysis by combining 22 newly sequenced fecal 
metagenomes of individuals from four countries with previously published data sets. They identified three robust clusters of gut bacteria (referred to as enterotypes) that were not nation- or continent-specific [33]. These enterotypes were identified by the variation at the level of one of the three following genera: Bacteroides, Prevotella, and Ruminococcus. These enterotypes were not correlated with the BMI, age, gender, or nationality of the subjects studied. However, definition of the microbial species more often present in people who develop adenomas and carcinomas (other than those specifically on the adenoma or carcinoma), compared with normal healthy people, has not yet been done. The Human Microbiome Project [34•, 35] has defined the normal fecal flora in more than 200 healthy men and women younger than aged 45 years. In that study, the major genera using the relatively new technique of 16S Ribosomal RNA typing was shown to be Bacteroides followed by Prevotella (both gram-negative bacteria). In those individuals with low Bacteroides, Fermicutes (gram-positive bacteria) was the major phylum present.

In a subsequent study published in Science, $\mathrm{Wu}$ and colleagues performed investigations into the relationship between diet and prevalence of enterotype using healthy volunteers $[36 \bullet \bullet]$. Using food-frequency questionnaires and obtaining stool samples from the 98 volunteers from which they could analyze DNA content to provide a snapshot of the gut microbiota, the authors showed that the Bacteroides enterotype was more common among individuals who reported eating a lot of meat and saturated fat, whereas in those who reported eating diets that were rich in carbohydrate, Prevotella was the dominant enterotype. In individuals consuming alcohol and high intakes of polyunsaturated fat, Ruminococus was the most common enterotype. Wu and colleagues then performed a controlled-feeding study of ten subjects and showed that the composition of the microbiome changed within 24 hours after initiating a high-fat/low-fiber or low-fat/high-fiber diet, but that the enterotype identity remained stable during the 10 -day study [36••]. These data suggest that the composition of the microbiome is influenced by diet and that it may be feasible to alter the enterotype through long-term dietary manipulation.

\section{Obesity and the Microbiome and Carcinogenesis}

Obesity is clearly linked to colorectal cancer [5•], and this link may be via the postulated differences in Bacteroides and Firmicutes between lean and obese subjects [35]. However, other investigators have found no differences in the microbiome between lean and obese subjects [37], and no changes with weight loss. It is possible that obesity could be linked to colorectal cancer via lower levels of bifidobacteria, which has been noted in obese women (who have higher levels of Bacteroides, Clostridium, and Staphylococcus) who gain excessive weight during pregnancy and also in people with type 2 diabetes-itself a putative risk factor for colorectal cancer [5•, 38, 39]. Weight loss also has been associated with reduced level of Bifidobacterium bifidum and Bifidobacterium breve counts and increased Bifidobacterium catenulatum [40]. The level of Bifidobacterium genus also is decreased upon weight loss after bariatric surgery [41].

Although there is a large amount of mouse data, there are little human data on the possible interactions between obesity, the microbiome, and the response to different macronutrients, including fiber. It has been shown that an increase in calorie intake (from 2,400 to 3,400 kcal/d) in obese and lean human individuals promotes rapid changes in the gut microbiota (20\% increase in Firmicutes and a corresponding decrease in Bacteroidetes) [42]. This was associated with an increased energy harvest of $\approx 150 \mathrm{kcal}$; the overfeeding in lean individuals was accompanied by a greater fractional decrease in stool energy loss [42]. The controversial research pertaining to the relationship between obesity and the microbiome has been extensively reviewed by Delzenne [43].

\section{Effects of Nonabsorbable Carbohydrates on the Microbiota}

Prebiotics are nondigested carbohydrates, which alter gut bacteria amounts, types, and activities in a way that is presumed (but not proven) to be favorable. In general, after prebiotics, bifidobacteria and lactobacilli increase and clostridia and coliforms decrease, but whether these bacteria are related to colorectal cancer is not known [44]. Weickert and colleagues [45] contrasted $43 \mathrm{~g}$ of fiber with $14 \mathrm{~g}$ of fiber (mostly cellulose and hemicellulose, and thus poorly fermentable) over 18 weeks in 69 people and measured butyrate producing species: no changes in species were seen with fiber, suggesting that at least for nonfermentable fiber, its potential protection from colorectal cancer is not via species change.

Walker and colleagues [46] performed a controlled feeding study in six overweight men with resistant starch (RS), nonstarch polysaccharide (NSP) and a reduced carbohydrate weight loss diet over 10 weeks to examine stool 16s RNA sequences; 320 phylotypes were identified. Changes in bacterial groups occurred with diet but there was marked individual variation and samples clustered more by individual than by diet. Relatives of Ruminococcus bromii increased in most volunteers on the RS diet, accounting for a mean of $17 \%$ of total bacteria compared with $3.8 \%$ on the NSP diet, whereas the Oscillibacter group increased on the RS and weight loss diets. Relatives of Eubacterium rectale increased on RS (to mean $10.1 \%$ ) but decreased on the weight loss diet. Interindividual variation was marked, however, with $>60 \%$ of RS remaining unfermented in two volunteers on the RS diet, compared with $<4 \%$ in the other 12 
volunteers; these two individuals also showed low numbers $(<1 \%)$ of Ruminococci.

Fructo-oligosaccharides (FOS), galacto-oligosaccharides (GOS), and lactulose are readily fermented and selectively stimulate particular species of bacteria usually bifido bacteria [44]. Consumption of 5-10 g of GOS led to five- to tenfold increases in bifidobacteria in half of the subjects with decreases in Bacteroidetes. Increases in Firmicutes also were observed in a few individuals. The responses to GOS and the magnitude of the response varied between individuals were reversible and were dose-related [47]. GOS enriches different lineages within the genus Bifidobacterium compared with resistant starches [48]. RS4 (chemically modified starch) but not RS2 (resistant granules) significantly increased Actinobacteria and Bacteroidetes while decreasing Firmicutes. RS4 increased Bifidobacterium adolescentis (in some subjects up to $18-30 \%$ of the population) and Parabacteroides distasonis, whereas RS2 increased Ruminococcus bromii and Eubacterium rectale compared with RS4. The responses to resistant starch and the size of the change varied between individuals. The changes were reversible and associated with the consumption of RS.

\section{The Butyrate Hypothesis and Risk of Colorectal Carcinogenesis}

The effects of butyrate on slowing the growth of colon cancer cells lines was first described in 1980 [49]. There have been more than 600 publications on butyrate since then, mostly in experimental animals and in vitro systems. In humans, there is no known evidence that butyrate levels or production and fecal $\mathrm{pH}$ is different in those with and without colorectal cancer $[50,51]$. A recent review examined the potential mechanisms of butyrate protection from colorectal carcinogenesis [52]. Butyrate slows cancer cell proliferation and induces differentiation and apoptosis, via inhibition of histone deactylase, but also inactivates carcinogens, through glutathione-S transferase enzyme induction. The mechanism of the GST induction may be via histone deactylase inhibition and modulation of MAPK pathways through ERK phosphorylation. Butyrate induces the phase 2 enzyme Glutathione S-transferase and NAD(P) $\mathrm{H}$ :quinone oxidoreductase (NQO) in a dose-dependent manner in rat intestinal cells. This was related to an increase of NF-E2-related factor 2 (Nrf2) nuclear translocation and a decrease in the levels of nuclear fraction p53 mRNA [53].

Butyrate signals via gut $G$ protein coupled receptors GPR41, GPR43, and GPR109A the expression of which is frequently lost in colonic cancer cells [54]. Apoptosis induced by activation of GPR109A with butyrate in colon cancer cells does not involve inhibition of histone deacetylation. The primary changes in this apoptotic process include down-regulation of $\mathrm{Bcl}-2, \mathrm{Bcl}-\mathrm{xL}$, and cyclin D1 and up-regulation of death receptor pathways. In addition, GPR109A/butyrate suppresses nuclear factor-kappaB activation in normal and cancer colon cell lines as well as in normal mouse colon. GPR109A is silenced in colon cancer cells [55].

Germ-free animals have low levels of GPR109A, a butyrate receptor, and SLC5A8, a butyrate transporter in the ileum and the colon. The expression returns to normal levels when germ-free mice are colonized with bacteria. Microarray analysis identifies approximately 700 gut genes whose expression is altered more than twofold in germ-free mice compared with conventional mice. Among these genes are the chloride/bicarbonate exchanger SLC26A3 and the water channel aquaporin 4 [56].

Increasing butyrate by attaching it to resistant starch in the colon of AOM-treated rats increases apoptosis by $50-70 \%$ 6 hours after AOM [57], resulting in lower large bowel tumor numbers after 25 weeks [58]. Resistant starch without added butyrate lowered tumor numbers but did not significantly change apoptosis rates, possibly suggesting different mechanisms of protection. Rates of apoptosis were not increased by red meat or increased amounts of protein but were lower with resistant starch (presumably reflecting less DNA damage) and protein fermentation products (fecal ammonia and phenol) also were reduced in mice fed resistant starch reflecting diversion of these components into microbial synthesis stimulated by carbohydrate [59].

Red meat in mice increases the promutagenic adduct $\mathrm{O}$ (6)-methyl-2-deoxyguanosine in colonocytes compared with casein, although there was no dose effect on either diet, whereas mice fed $10 \%$ high amylose starch had reduced levels of the adduct and increased short chain fatty acids. These results differ from the results seen with the comet assay in rats where increasing casein and soy from $15 \%$ to $25 \%$ increases DNA damage, which is partially reversed by a very high resistant starch diet (48 \% high amylose starch) but the effects on DNA damage were unrelated to fecal short chain fatty acids [60]. High amylose starch with butyrate attached increased fecal butyrate and was twice as effective as normal high amylose starch at reversing high protein-induced DNA damage. High protein diets on a background of low-amylose wheat or a novel high amylose wheat diet did not increase DNA damage [61].

\section{Impact of Weight Loss}

Weight loss diets that have reduced carbohydrate and increased protein are speculated potentially to increase the risk of colorectal cancer (despite the likely protective effect conferred by weight loss itself) by lowering butyrate concentrations [62] and lowering the number of butyrate producing bacteria [63] and increasing the proportions of branched-chain fatty acids and concentrations of phenylacetic acid and 
$\mathrm{N}$-nitroso compounds. Very low carbohydrate diets $(22 \mathrm{~g} / \mathrm{d})$ in 17 obese men for 4 weeks reduced the Roseburia/Eubacterium rectale group of bacteria (Firmicutes phylum, Lachnospiracea family) and greatly reduced concentrations of fiberderived, antioxidant phenolic acids, such as ferulate and its derivatives [64] compared with high carbohydrate diets $(360 \mathrm{~g} / \mathrm{d})$. There was no significant change in the overall proportion of Lachnospiraceae or in a second major group of butyrate-producing gram-positive bacteria related to Faecalibacterium prausnitzii. The proportion of Bacteroides spp. decreased by $22 \%$ with the very low carbohydrate diet compared with that with high carbohydrate diets and the total number of bacteria was greater $(P<0.012)$ with the high carbohydrate diet than with the other two diets with lower amounts of carbohydrate.

In summary, the proposition that differences in microbial population underlie population differences in colorectal cancer was extensively investigated in the 1970s, without a clear outcome because of problems with culturing many species. This question is now being addressed with molecular techniques; at present the only data show differences in the microbiome adherent to adenomas [65] and carcinomas $[66,67]$, although this may have nothing to do with causation and may merely reflect changes in mucin layers in the tumors. It is not clear if luminal or mucosal bacteria (or both) are the important players in carcinogenesis. Nor is it clear that bacteria in the Bacteroidetes phylum are beneficial and those in the Fermicutes phylum are harmful, as butyrate-producing species are found in both, whereas bifidobacteria may be helpful and lactobacilli may be harmful. Weight loss appears to reduce some butyrate-producing species. Moreover, the response of the microbiota to different forms of fiber are very variable and, if protection from colorectal cancer is related to shifts in microbial populations with fiber, then only a small proportion of the population on a high fermentable fiber diet will gain protection, thus diluting the overall effect seen in a general population study.

\section{Conclusions}

In 2011, in the wake of findings from several, large, prospective, epidemiological studies, the WCRF revised their consensus statement of a "probable" protective effect of dietary fiber on subsequent risk of developing colorectal cancer. This scientific body now considers the inverse association between fiber intake and risk of colorectal cancer to be supported by a "convincing" level of evidence. The magnitude of the association is comparable to that reported for physical activity, the only other modifiable lifestyle for which there is strong epidemiological evidence of a protective effect against developing this malignancy. However, uncertainty remains regarding the specific impact of dietary fiber on subsites of colorectal cancer and whether the protective effect of fiber on subsequent risk of the malignancy is apparent irrespective of its dietary source. Moreover, the biological mechanisms by which dietary fiber exerts its anticarcinogenic effects have yet to be fully elucidated, although there have been some recent exciting developments relating to the role of the microbiome. Nevertheless, the data are sufficiently robust to warrant the incorporation of the recommendation for increasing total dietary fiber intake alongside those advocating increased physical activity public health guidelines for the primary prevention of this highly preventable malignancy.

Acknowledgments M. Woodward is supported by a grant from Roche for the ACTION Study.

Disclosure No potential conflicts of interest relevant to this article were reported.

\section{References}

Papers of particular interest, published recently, have been highlighted as:

- Of importance

•- Of major importance

1. Stewart BW, Kleihues P, editors. World cancer report. Lyon: IARC Press; 2003.

2. Parkin DM, Bray F, Ferlay J, et al. Global cancer statistics, 2002. CA Cancer J Clin. 2005;55:74-108.

3. Schottenfeld D, Fraumeni F, editors. Cancer epidemiology and prevention. 3rd ed. New York: Oxford University Press; 2006.

4. McCredie M, Williams S, Coates M. Cancer mortality in migrants from the British Isles and continental Europe to New South Wales, Australia, 1975-1995. Int J Cancer. 1999;83:179-85.

5. • Huxley RR, Ansary-Moghaddam A, Clifton P, et al. The impact of dietary and lifestyle risk factors on risk of colorectal cancer: a quantitative overview of the epidemiological evidence. Int J Cancer. 2009;125:171-80. This article provides a quantitative summary of diet and lifestyle risk factors for colorectal cancer.

6. Terry P, Giovannucci E, Michels KB, et al. Fruit, vegetables, dietary fiber, and risk of colorectal cancer. J Natl Cancer Inst. 2002;93:525-33.

7. Gonzalez CA. Nutrition and cancer: the current epidemiological evidence. Br J Nutr. 2006;96:42-5.

8. Samad AK, Taylor RS, Marshall T, et al. A meta-analysis of the association of physical activity with reduced risk of colorectal cancer. Colorectal Dis. 2005;7:204-13.

9. Bianchini F, Kaaks R, Vainio H. Overweight, obesity, and cancer risk. Lancet Oncol. 2002;3:565-74.

10. Larsson SC, Orsini N, Wolk A. Diabetes mellitus and risk of colorectal cancer: a meta-analysis. J Natl Cancer Inst. 2005;97:1679-87.

11. Potter JD, Slattery ML, Bostick RM, et al. Colon cancer: a review of the epidemiology. Epidemiol Rev. 1993;15:499-545.

12. World Cancer Research Fund / American Institute for Cancer Research. Food, nutrition, physical activity, and the prevention of cancer: a global perspective. Washington: AICR; 2007. 
13. Bingham SA, Norat T, Moskal A, et al. Is the association with fiber from foods in colorectal cancer confounded by folate intake? Cancer Epidemiol Biomarkers Prev. 2005;14:1552-6.

14. Park Y, Hunter DJ, Spiegelman D, et al. Dietary fiber intake and risk of colorectal cancer. JAMA: The Journal of the American Medical Association. 2005;294:2849-57.

15. Dietary Reference Intakes for Energy, Carbohydrate, fiber, Fat, Fatty Acids, Cholesterol, Protein, and Amino Acids (Macronutrients) (2005) Chapter 7: Dietary, Functional and Total Fiber." U.S. Department of Agriculture, National Agricultural Library and National Academy of Sciences, Institute of Medicine, Food and Nutrition Board.

16. Shin A, Li H, Shu XO, et al. Dietary intake of calcium, fiber and other micronutrients in relation to colorectal cancer risk: results from the Shanghai Women's Health Study. Int J Cancer. 2006;119:2938-42.

17. Otani T, Iwasaki M, Ishihara J, et al. Dietary fiber intake and subsequent risk of colorectal cancer: the Japan Public Health Center-based prospective study. Int J Cancer. 2006;119:1475-80.

18. McCarl M, Harnack L, Limburg PJ, et al. Incidence of colorectal cancer in relation to glycemic index and load in a cohort of women. Cancer Epidemiol Biomarkers Prev. 2006;15:892-6.

19. Wakai K, Date C, Fukui M, et al. Dietary fiber and risk of colorectal cancer in the Japan collaborative cohort study. Cancer Epidemiol Biomarkers Prev. 2007;16:668-75.

20. Schatzkin A, Mouw T, Park Y, et al. Dietary fiber and whole-grain consumption in relation to colorectal cancer in the NIH-AARP diet and health study. Am J Clin Nutr. 2007;85:1353-60.

21. Nomura AM, Hankin JH, Henderson BE, et al. Dietary fiber and colorectal cancer risk: the multiethnic cohort study. Cancer Causes Control. 2007;18:753-64.

22. Kabat GC, Shikany JM, Beresford SA, et al. Dietary carbohydrate, glycemic index, and glycemic load in relation to colorectal cancer risk in the Women's Health Initiative. Cancer Causes Control. 2008;19:1291-8.

23. Butler LM, Wang R, Koh WP, Yu MC. Prospective study of dietary patterns and colorectal cancer among Singapore Chinese. Br J Cancer. 2008;99:1511-6.

24. •- Aune D, Chan DS, Lau R, et al. Dietary fiber, whole grains, and risk of colorectal cancer: systematic review and dose-response meta-analysis of prospective studies. BMJ. 2011;343:d6617. This article provides a quantitative summary of all published studies on the relationship between fiber and risk of colorectal cancer.

25. - Murphy N, Norat T, Ferrari P, et al. Dietary fiber intake and risks of cancers of the colon and rectum in the European prospective investigation into cancer and nutrition (EPIC). PLoS One. 2012;7: e39361. This article reports on the most recent findings from the largest multi-center cohort study on the relationship between types of dietary fiber and colorectal cancer risk.

26. • World Cancer Research Fund / American Institute for Cancer Research. Continuous Update Project Report Summary. Food, Nutrition, Physical Activity, and the Prevention of Colorectal Cancer. 2011. This report is a detailed examination of all risk factors for colorectal cancer.

27. Bingham SA. Mechanisms and experimental and epidemiological evidence relating dietary fiber (non-starch polysaccharides) and starch to protection against large bowel cancer. Proc Nutr Soc. 1990;49:153-71.

28. Ottman N, Smidt H, de Vos WM, Belzer C. The function of our microbiota: who is out there and what do they do? Front Cell Inf Microbiol. 2012;2:104.

29. Van Tassell RL, Kingston DG, Wilkins TD. Metabolism of dietary genotoxins by the human colonic microflora; the fecapentaenes and heterocyclic amines. Mutat Res. 1990;238:209-21.

30. de Kok TM, van Maanen JM. Evaluation of fecal mutagenicity and colorectal cancer risk. Mutat Res. 2000;463:53-101.
31. Reddy BS, Weisburger JH, Narisawa T, Wynder EL. Colon carcinogenesis in germ-free rats with 1,2-dimethylhydrazine and N-methyl-n'-nitro-N-nitrosoguanidine. Cancer Res. 1974;34:2368-72.

32. Hebels DG, Sveje KM, de Kok MC, et al. Red meat intake-induced increases in fecal water genotoxicity correlate with procarcinogenic gene expression changes in the human colon. Food Chem Toxicol. 2012;50:95-103. Epub 2011 Oct 14.

33. Arumugam M, Raes J, Pelletier E, et al. Enterotypes of the human gut microbiome. Nature. 2011;473:174-80.

34. - Human Microbiome Project Consortium. Structure, function and diversity of the healthy human microbiome. Nature. 2012;13 (486):207-14. This article provides information about the study design, objectives, and expected outcomes for this international collaboration.

35. Segata N, Haake SK, Mannon P, et al. Composition of the adult digestive tract bacterial microbiome based on seven mouth surfaces, tonsils, throat and stool samples. Genome Biol. 2012;13:R42.

36. •• Wu GD, Chen J, Hoffmann C, et al. Linking long-term dietary patterns with gut microbial enterotypes. Science. 2011;334:105-8. This article reports on the impact of dietary manipulation on the composition of the microbiome.

37. Duncan SH, Lobley GE, Holtrop G, et al. Human colonic microbiota associated with diet, obesity and weight loss. Int J Obes (Lond). 2008;32:1720-4.

38. Collado MC, Isolauri E, Laitinen K, Salminen S. Distinct composition of gut microbiota during pregnancy in overweight and normal-weight women. Am J Clin Nutr. 2008;88:894-9.

39. Wu X, Ma C, Han L, et al. Molecular characterisation of the faecal microbiota in patients with type II diabetes. Curr Microbiol. 2010;61:69-78.

40. Santacruz A, Marcos A, Warnberg J, et al. Interplay between weight loss and gut microbiota composition in overweight adolescents. Obesity. 2009;17:1906-15.

41. Furet JP, Kong LC, Tap J, et al. Differential adaptation of human gut microbiota to bariatric surgery-induced weight loss: links with metabolic and low-grade inflammation markers. Diabetes. 2010;59:3049-57.

42. Jumpertz R, Le DS, Turnbaugh PJ, et al. Energy-balance studies reveal associations between gut microbes, caloric load, and nutrient absorption in humans. Am J Clin Nutr. 2011;94:58-65.

43. Delzenne NM, Cani PD. Interaction between obesity and the gut microbiota: relevance in nutrition. Annu Rev Nutr. 2011;31:15-31.

44. Roberfroid M, Gibson GR, Hoyles L, et al. Prebiotic effects: metabolic and health benefits. Br J Nutr. 2010;104(2):S1-63.

45. Weickert MO, Arafat AM, Blaut M, et al. Changes in dominant groups of the gut microbiota do not explain cereal-fiber induced improvement of whole-body insulin sensitivity. Nutr Metab. 2011;8:90.

46. Walker AW, Ince J, Duncan SH, et al. Dominant and dietresponsive groups of bacteria within the human colonic microbiota. ISME J. 2011;5:220-30.

47. Davis LM, Martínez I, Walter J, Goin C, Hutkins RW. Barcoded pyrosequencing reveals that consumption of galactooligosaccharides results in a highly specific bifidogenic response in humans. PLoS One. 2011;6:e25200.

48. Martínez I, Kim J, Duffy PR, Schlegel VL, Walter J. Resistant starches types 2 and 4 have differential effects on the composition of the fecal microbiota in human subjects. PLoS One. 2010;5:e15046.

49. Kim YS, Tsao D, Siddiqui B, Whitehead JS, Arnstein P, Bennett J, Hicks J. Effects of sodium butyrate and dimethylsulfoxide on biochemical properties of human colon cancer cells. Cancer. 1980;45(5 Suppl):1185-92.

50. Brinkworth GD, Noakes M, Clifton PM, Bird AR. Comparative effects of very low-carbohydrate, high-fat and high-carbohydrate, 
low-fat weight-loss diets on bowel habit and faecal short-chain fatty acids and bacterial populations. Br J Nutr. 2009;101:1493-502.

51. Duncan SH, Belenguer A, Holtrop G, et al. Reduced dietary intake of carbohydrates by obese subjects results in decreased concentrations of butyrate and butyrate-producing bacteria in feces. Appl Environ Microbiol. 2007;73:1073-8.

52. Russell WR, Gratz SW, Duncan SH, et al. High-protein, reducedcarbohydrate weight-loss diets promote metabolite profiles likely to be detrimental to colonic health. Am J Clin Nutr. 2011;93:1062-72.

53. Yaku K, Enami Y, Kurajyo C, et al. The enhancement of phase 2 enzyme activities by sodium butyrate in normal intestinal epithelial cells is associated with Nrf2 and p53. Mol Cell Biochem. 2012 Jul 18. [Epub ahead of print]

54. Tang Y, Chen Y, Jiang H, Robbins GT, Nie D. G-protein-coupled receptor for short-chain fatty acids suppresses colon cancer. Int $\mathrm{J}$ Cancer. 2011;128:847-56.

55. Thangaraju M, Cresci GA, Liu K, et al. GPR109A is a Gprotein-coupled receptor for the bacterial fermentation product butyrate and functions as a tumor suppressor in colon. Cancer Res. 2009;69:2826-32.

56. Cresci GA, Thangaraju M, Mellinger JD, Liu K, Ganapathy V. Colonic gene expression in conventional and germ-free mice with a focus on the butyrate receptor GPR109A and the butyrate transporter SLC5A8. J Gastrointest Surg. 2010;14:449-61.

57. Clarke JM, Young GP, et al. Butyrate delivered by butyrylated starch increases distal colonic epithelial apoptosis in carcinogentreated rats. Carcinogenesis. 2012;33:197-202.

58. Clarke JM, Topping DL, Bird AR, Young GP, Cobiac L. Effects of high-amylose maize starch and butyrylated high-amylose maize starch on azoxymethane-induced intestinal cancer in rats. Carcinogenesis. 2008;29:2190-4.

59. Winter J, Nyskohus L, Young GP, et al. Inhibition by resistant starch of red meat-induced promutagenic adducts in mouse colon. Cancer Prev Res (Phila). 2011;4:1920-8.

60. Toden S, Bird AR, Topping DL, Conlon MA. High red meat diets induce greater numbers of colonic DNA double-strand breaks than white meat in rats: attenuation by high-amylose maize starch. Carcinogenesis. 2007;28:2355-62.

61. Conlon MA, Kerr CA, McSweeney CS, et al. Resistant starches protect against colonic DNA damage and alter microbiota and gene expression in rats fed a Western diet. J Nutr. 2012;142:832-40.

62. Clausen MR, Bonnén H, Mortensen PB. Colonic fermentation of dietary fiber to short chain fatty acids in patients with adenomatous polyps and colonic cancer. Gut. 1991;32:923-8.

63. Hove H, Rye Clausen M, Brøbech Mortensen P. Lactate and $\mathrm{pH}$ in faeces from patients with colonic adenomas or cancer. Gut. 1993;34:625-9.

64. Fung KY, Cosgrove L, Lockett T, Head R, Topping DL. A review of the potential mechanisms for the lowering of colorectal oncogenesis by butyrate. Br J Nutr. 2012;7:1-12.

65. Sanapareddy N, Legge RM, Jovov B, et al. Increased rectal microbial richness is associated with the presence of colorectal adenomas in humans. ISME J. 2012 May 24.

66. Marchesi JR, Dutilh BE, Hall N, et al. Towards the human colorectal cancer microbiome. PLoS One. 2011;6:e20447.

67. Sobhani I, Tap J, Roudot-Thoraval F, et al. Microbial dysbiosis in colorectal cancer (CRC) patients. PLoS One. 2011;6:e16393. 